\title{
Hamilton-Jacobi treatment of a non-relativistic particle on a curved space
}

\author{
Dumitru Baleanu[ and Yurdahan Güler" \\ Department of Mathematics and Computer Sciences, Faculty of Arts and Sciences, \\ Cankaya University-06530, Ankara, Turkey
}

\begin{abstract}
In this paper a non-relativistic particle moving on a hypersurface in a curved space and the multidimensional rotator are investigated using the Hamilton-Jacobi formalism. The equivalence with the Dirac Hamiltonian formalism is demonstrated in both Cartesian and curvilinear coordinates. The energy spectrum of the multidimensional rotator is equal to that of a pure Laplace-Beltrami operator with no additional constant arising from the curvature of the sphere.
\end{abstract}

\footnotetext{
${ }^{1}$ On leave of absence from Institute of Space Sciences, P.O.BOX, MG-23, R 76900, MagureleBucharest, Romania, E-mail: baleanu@venus.nipne.ro

${ }^{2}$ E-Mail: yurdahan@cankaya.edu.tr
} 


\section{Introduction}

Constraint Hamiltonian systems play a crucial role in gauge theories. Since Dirac's pioneering work [1] on constrained systems, there has been considerable progress in this field [2, 3, 4, 5]. Although some basic steps were taken, there are still some more problems which need deeper analysis. Especially one should define the Dirac brackets explicitly to quantize second-class constraints. But this is not an easy task, because except for very particular cases, e.g. the Dirac brackets are c-numbers, this problem does not have a general solution. In other words, it is extremely difficult to find a representation for the independent operators.Determination of degre of freedom of a singular system is a vital problem especially if second- class constraints exist. In fact, the reduced phase space is a symplectic manifold in mathematical language [6] and the Darboux theorem ensures that one can find, at least locally, the coordinates in terms of which the Poisson brackets (defined on the reduced phase space in the presence of constraints) have the canonical form.

Quantization of a free point particle in curved space is a long-standing and controversial problem in quantum mechanics [7, 8]. Dirac has emphasized that canonical quantization rules are consistent only in a Cartesian reference frame. Attempts to generalize these rules to curved space run into the notorious operator-ordering problem of momentum and coordinates [1]. Podolski avoided this problem [9] by postulating that the Laplacian in the free Schrödinger operator $H=-\frac{\hbar^{2} \triangle}{2}$ should be replaced by the Laplace-Beltrami operator $\triangle_{L B}=g^{\frac{-1}{2}} \partial_{\mu} g^{\frac{1}{2}} g^{\mu \nu} \partial_{\nu}$, where $\partial_{\mu}=\frac{\partial}{\partial q^{\mu}}$ are partial derivatives with respect to the $\mathrm{N}$ - dimensional curved space coordinates, and $\mathrm{g}$ is the determinant of the metric $g_{\mu \nu}(q)$. This postulate has generally been accepted as being correct since it yields, for a N-dimensional Cartesian space with coordinates $x^{i}$, an energy $\frac{\hat{L}_{a}^{2}}{2 R^{2}}$. Here $\hat{L}_{a}=-i \hat{p}_{i}\left(L_{a}\right)_{i j} x_{j}$ with $\hat{p}_{i}=-i \hbar \frac{\partial}{\partial x_{i}}$ are the unique quantum-mechanical differential operator representation of the $\frac{N(N+1)}{2}$ generators $L_{a}$ of the rotation group $S O(N+1)$ in flat space [10. A discrepancy with the Dirac formalism has however been reported in several papers [8, 10, 11], as well as other different results [12, 13]. In spite of all these developments, the status of the problem is very confusing, and there have been many papers claiming a rejection of the Dirac formalism [10], an intrinsic difference between path formulation and operator formalism [11], or advocating different quantization schemes [12]. Besides, in the definition of the Wheeler-de-Witt equation [14], which has the central importance in string theories, in curved space this problem arises again.

An alternative method of quantization is the Hamilton-Jacobi formulation initiated by one of us 15, 16, 17]. Using the Carathéodory's equivalent Lagrangian method we find a set of Hamilton-Jacobi equations integrated by the method of characteristics [18, 19]. Recently this formalism was generalized to the singular systems with higher order Lagrangians and to systems which have elements of the Berezin algebra [20, 21, 22]. Even more recently the quantization of the systems with constraints was investigated using this approach [23, 24, 25]. The advantage of using the Hamilton-Jacobi formalism is that we have no difference between first and second class constraints and we do not need gauge 
fixing term because the gauge variables are separated in the processs of constructing an integrable system of total differential equations. In addition the action provided by the formalism can be used in the process of path integral quantization method of the constrained systems. However, the quantization of the systems with second class constraints is problematic for Hamilton-Jacobi formalism because the system of equations is not integrable. To solve this problem we have two basic possibilities ,the first one is to enlarge the phase space [26] and the other one is to keep to the original phase space itself [27, 28].

Let us consider a singular Lagrangian with Hessian matrix of rank n-r . The formalism leads us to the following Hamiltonians

$$
H_{\alpha}^{\prime}=H_{\alpha}\left(t_{\beta}, q_{a}, p_{a}\right)+p_{\alpha}
$$

where $\alpha, \beta=n-r+1, \cdots, n, a=1, \cdots n-r$. The usual hamiltonian $H_{0}$ is defined as

$$
H_{0}=-L\left(t, q_{i}, \dot{q}_{\nu}, \dot{q}_{a}=w_{a}\right)+p_{a} w_{a}+\left.\dot{q}_{\mu} p_{\mu}\right|_{p_{\nu}=-H_{\nu}}, \nu=0, n-r+1, \cdots, n
$$

which is independent of $\dot{q}_{\mu}$. Here $\dot{q}_{a}=\frac{d q_{a}}{d \tau}$, where $\tau$ is a parameter and $\omega_{a}$ are obtained from the definition of generalized momenta. The equations of motion are obtained as total differential equations in many variables as follows

$$
\begin{gathered}
d q_{a}=\frac{\partial H_{\alpha}^{\prime}}{\partial p_{a}} d t_{\alpha}, d p_{a}=-\frac{\partial H_{\alpha}^{\prime}}{\partial q_{a}} d t_{\alpha}, d p_{\mu}=-\frac{\partial H_{\alpha}^{\prime}}{\partial t_{\mu}} d t_{\alpha}, \mu=1, \cdots, r, \\
d z=\left(-H_{\alpha}+p_{a} \frac{\partial H_{\alpha}^{\prime}}{\partial p_{a}}\right) d t_{\alpha},
\end{gathered}
$$

where $z=S\left(t_{\alpha}, q_{a}\right)$ is the Hamilton-Jacobi function.

One should notice that although we have started with $n$ generalized coordinates $q_{i}$ and generalized velocities $\dot{q}_{i}$ to pass to canonical formulation we have to treat some generalized momenta dependent and corresponding generalized coordinates as free parameters. Thus , we have a phase space of lower dimension. But this is not sufficient simply because that the equations of motion are total differential equations and we should consider integrability conditions. In other words eqs. (3, 1 ) are integrable iff $d H_{\alpha}{ }^{\prime}=0$. Some of these conditions could be satisfied identically and the rest may cause new constraints.Again using the same test the additional constraints, might arise. As a result, it may happen that we have a set of constraints which are in involution and an integrable system. Every new constraint causes to reduce the dimension of the phase space. In the end we may have constraints in the form

$$
H_{0}^{\prime}=p_{0}+H_{0}, H_{\gamma}^{\prime}=H_{\gamma}\left(t_{\beta}, q_{a}, p_{a}\right)+p_{\gamma}
$$

and additional constraints which can not be expressed in this form.

The equations of motion take the form

$$
d q_{b}=\frac{\partial H_{0}^{\prime}}{\partial p_{b}} d \tau+\frac{\partial H_{\gamma}^{\prime}}{\partial p_{b}} d t_{\gamma}
$$




$$
d p_{b}=-\frac{\partial H_{0}^{\prime}}{\partial q_{b}} d \tau-\frac{\partial H_{\gamma}^{\prime}}{\partial q_{b}} d t_{\gamma}
$$

Thus, we have an integrable system with some additional constraints.

The action can be obtained solving the following equation by quadratures:

$$
d z=\left(-H_{0}+p_{a} \frac{\partial H_{0}^{\prime}}{\partial p_{a}}\right) d \tau+\left(-H_{\beta}+p_{a} \frac{\partial H_{\beta}^{\prime}}{\partial p_{a}}\right) d t_{\beta} .
$$

This paper is organized as follows.

In Section 2 the non-relativistic particle moving on a hypersurface in a curved manifold is investigated using the Hamilton-Jacobi formalism. The multidimensional rotator is analyzed and the results are compared with those obtained by the Dirac Hamiltonian formalism. In Section 3 the quantization of the multidimensional rotator is investigated. In Section 4 concluding remarks are presented.

\section{Hamilton-Jacobi formalism of the non-relativistic particle moving on a hypersurface in a curved man- ifold}

We consider a n-dimensional manifold equipped with the Riemannian metric $g_{i j}(x)$. Let $x^{i}(i=1,2, \cdots n)$ be the coordinates of the manifold. We consider a non-relativistic particle of mass $m$ whose motion is constrained on the hypersurface defined as [1]

$$
f(x)=B,(B=\text { const }) .
$$

In the presence of the vector and scalar potentials $A_{i}(x)$ and $V(x)$, the Lagrangian is given by

$$
L=\frac{1}{2} g_{i j} \dot{x}^{i} \dot{x}^{j}+A_{i} \dot{x}^{i}-V(x)+\dot{\lambda}(f(x)-B) .
$$

Here $\dot{x}^{i}=\frac{d x^{i}}{d t}$ and $x^{i}(t)$ denotes the position of the particle , $\lambda$ is a Lagrange multiplier and $\dot{\lambda}=\frac{d \lambda}{d t}$. The canonical momenta conjugate to $x^{i}$ and $\lambda$ are

$$
p_{i}=m g_{i j} \dot{x}^{i}+A_{i}, p_{\lambda}=f(x)-B \text {. }
$$

This Lagrangian leads us to the following Hamiltonians

$$
\begin{aligned}
& H_{0}^{\prime}=p_{0}+\frac{1}{2 m} g^{i j}\left(p_{i}-A_{i}\right)\left(p_{j}-A_{j}\right)+V(x), \\
& H_{1}^{\prime}=p_{\lambda}-f(x)+B .
\end{aligned}
$$

The canonical equations are 


$$
\begin{aligned}
d x^{i} & =\frac{g^{i j}}{m}\left(p_{j}-A_{j}\right) d t \\
d p_{i} & =\left\{\frac{1}{2 m} \frac{\partial g^{l j}}{\partial x^{i}}\left(p_{l}-A_{i}\right)\left(p_{j}-A_{j}\right)+\frac{\partial V}{\partial x^{i}}-\frac{g^{l j}}{m} \frac{\partial A_{l}}{\partial x^{i}}\left(p_{j}-A_{j}\right)\right\} d t+\frac{\partial f}{\partial x^{i}} d \lambda \\
d p_{\lambda} & =0 .
\end{aligned}
$$

Imposing the variations of $(\sqrt{12})$ to be zero and take into account (13) we found immediately the consistency condition

$$
d f(x)=0
$$

Using (14) a new constraint arises

$$
H_{2}^{\prime}=\frac{g^{i j}}{m} \frac{\partial f}{\partial x^{i}}\left(p_{j}-A_{j}\right)
$$

Taking the variation of (15) and using (13) we obtain

$$
\begin{aligned}
& \frac{1}{m^{2}} \quad\left\{g^{k l}\left[\frac{\partial}{\partial x^{k}}\left(g^{i j} \frac{\partial f}{\partial x^{i}}\right) p_{j}-\frac{\partial}{\partial x^{k}}\left(g^{i j} \frac{\partial f}{\partial x^{i}} A_{j}\right)\right]\left(p_{l}-A_{l}\right)-\frac{1}{2} \frac{\partial g^{k l}}{\partial x^{i}} g^{i j} \frac{\partial f}{\partial x^{j}} p_{k} p_{l}\right. \\
& \left.+\quad \frac{\partial}{\partial x^{j}}\left(g^{k l} A_{l}\right) g^{i j} \frac{\partial f}{\partial x^{i}} p_{k}\right\}-\frac{1}{m} g^{i j} \frac{\partial f}{\partial x^{i}} \frac{\partial V}{\partial x^{j}}+\frac{g^{i j}}{m} \frac{\partial f}{\partial x^{i}} \frac{\partial f}{\partial x^{j}} \dot{\lambda}=0 .
\end{aligned}
$$

Solving (16) we find the Lagrange multiplier $\lambda$.

In order to compare our results with those obtained using Dirac's procedure we analyze the variations of $H_{0}^{\prime}, H_{1}^{\prime}, H_{2}^{\prime}$. Using

$$
\begin{aligned}
& d H_{1}^{\prime}=\left\{H_{0}^{\prime}, H_{1}^{\prime}\right\} d t, \\
& d H_{2}^{\prime}=\left\{H_{0}^{\prime}, H_{2}^{\prime}\right\} d t+\left\{H_{0}^{\prime}, H_{1}^{\prime}\right\} d \lambda
\end{aligned}
$$

we can prove easily that the integrability conditions of (13) are the same as Dirac's consistency conditions.

\subsection{Multidimensional rotator}

In order to clarify our method we will analyze in detail the multidimensional rotator problem. The Lagrangian for a particle of unit mass constrained to move on the surface of an $\mathrm{N}$-dimensional sphere of radius $\mathrm{R}$ is given by the well known expression

$$
L=\frac{1}{2} \dot{x}_{\alpha} \dot{x}^{\alpha}-\dot{\lambda}\left(x_{\alpha} x^{\alpha}-R^{2}\right), \alpha=1 \cdots N
$$

where the constraint

$$
f(x)=-x_{\alpha} x^{\alpha}+R^{2}=0
$$

is implemented by the Lagrangian multiplier $\dot{\lambda}$. Using (12), (13), (15) and (18) we get a new constraint

$$
x^{\alpha} p_{\alpha}=0
$$


and an equation for $\lambda$ as

$$
\dot{\lambda}=\frac{p_{\alpha} p^{\alpha}}{2 x_{\alpha} x^{\alpha}}
$$

Here $\lambda$ is a gauge parameter.To summarize we have the following set of Hamiltonians

$$
H_{0}^{\prime}=p_{0}+\frac{1}{2} p_{\alpha} p^{\alpha}, H_{1}^{\prime}=p_{\lambda}+x_{\alpha} x^{\alpha}-R^{2}, H_{2}^{\prime}=x^{\alpha} p_{\alpha}
$$

The transformation from Cartesian to curvilinear coordinates is defined as

$$
\begin{aligned}
x_{1} & =r \sin \varphi_{1} \cdots \sin \varphi_{N-1}, \quad x_{2}=r \sin \varphi_{1} \cdots \sin \varphi_{N-2} \cos \varphi_{N-1}, \\
x_{N-3} & =r \sin \varphi_{1} \sin \varphi_{2} \cos \varphi_{3}, \\
\cdots & =\cdots, \\
x_{N-1} & =r \sin \varphi_{1} \cos \varphi_{2}, \quad x_{N}=r \cos \varphi_{1} .
\end{aligned}
$$

In these new variables, the Lagrangian and the canonical momenta are given as

$$
\begin{gathered}
L=\frac{1}{2}\left(\dot{r}^{2}+r^{2} \dot{\varphi}_{1}^{2}+\cdots r^{2} \dot{\varphi}_{N-1}^{2} \cdots \sin ^{2} \varphi_{N-2}\right)+\dot{\lambda}(-r+R), \\
\pi_{\lambda}=-r+R \quad, \pi_{r}=\dot{r}, \pi_{\varphi_{1}}=r^{2} \dot{\varphi}_{1}, \pi_{\varphi_{2}}=r^{2} \sin ^{2} \varphi_{1} \dot{\varphi}_{2} \\
\cdots \\
\pi_{\varphi_{N-1}}=\quad, \quad r^{2} \sin ^{2} \varphi_{1} \cdots \sin ^{2} \varphi_{N-1} \dot{\varphi}_{N-1} .
\end{gathered}
$$

In the Hamilton-Jacobi formalism we have two Hamiltonians

$$
\begin{aligned}
H_{0}^{\prime} & =p_{0}+\frac{1}{2}\left(\pi_{r}^{2}+\frac{\pi_{\varphi_{1}}^{2}}{r^{2} \sin ^{2} \varphi_{1}}+\cdots+\frac{\pi_{\varphi_{N-1}}^{2}}{r^{2} \sin ^{2} \varphi_{1} \cdots r^{2} \sin ^{2} \varphi_{N-1}}\right), \\
H_{1}^{\prime} & =\pi_{\lambda}+r-R .
\end{aligned}
$$

Using the consistency conditions $d H_{0}^{\prime}=0$ and $d H_{1}^{\prime}=0$ from (13) we obtain

$$
d r=0, d \pi_{r}=0 .
$$

Taking into account (26) and (13) we have

$$
d \pi_{r}=-d \lambda+\frac{1}{r^{3}}\left(\frac{\pi_{\varphi_{1}}^{2}}{\sin ^{2} \varphi_{1}}+\cdots+\frac{\pi_{\varphi_{N-1}}^{2}}{\sin ^{2} \varphi_{1} \cdots \sin ^{2} \varphi_{N-1}}\right) d t .
$$

From (27) and (28) we find

$$
\dot{\lambda}=\frac{1}{r^{3}}\left(\pi_{r}^{2}+\frac{\pi_{\varphi_{1}}^{2}}{\sin ^{2} \varphi_{1}}+\cdots+\frac{\pi_{\varphi_{N-1}}^{2}}{\sin ^{2} \varphi_{1} \cdots \sin ^{2} \varphi_{N-1}}\right) .
$$

In general the physical variables are non-linear functions of the original variables of the system. The separation of local coordinates into the physical and pure gauge ones can 
be performed by choosing the curvilinear coordinates in such a way that some of them span gauge orbits, while the other change along the directions transverse to the gauge orbits and denote physical states ( for more details see Refs. [5, 29]). As an example we consider the three-dimensional case, in which the transformation from Cartesian to spherical coordinates is given as

$$
\begin{aligned}
x_{1} & =r \sin \theta \cos \phi, x_{2}=r \sin \theta \sin \varphi, \quad x_{3}=r \cos \theta, \\
\pi_{1} & =\sin \theta \cos \varphi \pi_{r}+r \cos \theta \cos \varphi \pi_{\theta}-r \sin \theta \sin \varphi \pi_{\varphi}, \\
\pi_{2} & =\sin \theta \sin \varphi \pi_{r}+r \cos \theta \sin \varphi \pi_{\theta}+r \sin \theta \cos \varphi \pi_{\varphi}, \\
\pi_{3} & =\cos \theta \pi_{r}-r \sin \theta \pi_{\theta}, \\
\lambda & =\lambda, \pi_{\lambda}=p_{\lambda} .
\end{aligned}
$$

This transformation is a canonical transformation [18, 19]. The canonical pairs are now well defined $\left(r, \pi_{r}\right),\left(\theta, \pi_{\theta}\right)$ and $\left(\phi, \pi_{\phi}\right)$.

The Hamiltonians have the following expressions

$$
\begin{aligned}
& H_{0}^{\prime}=p_{0}+\frac{\pi_{\theta}^{2}}{2 r^{2}}+\frac{\pi_{\varphi}^{2}}{2 r^{2} \sin ^{2} \theta}+\frac{\pi_{r}^{2}}{2}, \\
& H_{1}^{\prime}=\pi_{\lambda}+r-R
\end{aligned}
$$

and using (31) we obtain the following canonical equations

$$
\begin{aligned}
& d r=\pi_{r} d t, \quad d \theta=\frac{\pi_{\theta}}{r^{2}} d t, \quad d \varphi=\frac{\pi_{\varphi}}{r^{2} \sin ^{2} \theta} d t \\
& d \pi_{r}=-d \lambda+\frac{1}{r^{3}}\left(\pi_{\theta}^{2}+\frac{\pi_{\varphi}^{2}}{\sin ^{2} \theta}\right) d t, \quad d \pi_{\varphi}=0 \\
& d \pi_{\theta}=\frac{\pi_{\varphi}^{2}}{r^{2} \sin ^{3} \theta} \cos \theta d t \\
& d \pi_{\lambda}=0 .
\end{aligned}
$$

Imposing $d H_{0}^{\prime}=0$ and $d H_{1}^{\prime}=0$ we obtain $H_{2}^{\prime}=r \pi_{r}=0$. Taking into account the consistency condition obtained above and using (27) we find

$$
\dot{\lambda}=\frac{1}{r^{3}}\left(\pi_{\theta}^{2}+\frac{\pi_{\varphi}^{2}}{\sin ^{2} \theta}\right)
$$

In this case the action has the following expression

$$
z=\frac{1}{2 R^{2}} \int\left(\pi_{\theta}^{2}+\frac{\pi_{\varphi}^{2}}{\sin ^{2} \theta}\right) d t
$$

\section{Quantization of the multidimensional rotator}

The multidimensional rotator is a system having second-class constraints in Dirac's classification of the constrained systems. The Hamilton -Jacobi formalism leads us to three 
Hamiltonians $H_{0}^{\prime}, H_{1}^{\prime}, H_{2}^{\prime}$ which are not in involution. At this stage we mention that it is possible always to make the Hamltonians in involution and then the corresponding new system is integrable. In our specific problem we can apply the method of Abelian conversion to transform the system into an Abelian gauge theory [30].

We found the Hamiltonians in involution :

$$
H_{0}^{\prime \prime}=p_{0}+\frac{1}{2}\left(\frac{\left(H_{2}^{\prime \prime}\right)^{2}}{H_{1}^{\prime \prime}+R^{2}}+\frac{L_{a}^{2}}{H_{1}^{\prime \prime}+R^{2}}\right), H_{1}^{\prime \prime}=p_{\lambda}+x_{\alpha} x^{\alpha}-R^{2}, H_{2}^{\prime \prime}=x^{\alpha} p_{\alpha}+2 x^{2} \lambda,
$$

where $L_{a}=-i p L_{a} x$ is the classical component of the angular momentum (with $a=i, j$, $\left.L_{a}=x_{i} p_{j}-x_{j} p_{i}\right)$.

At the quantum level we obtain $H_{0}^{\prime \prime} \Psi=H_{2}^{\prime \prime} \Psi=H_{1}^{\prime \prime} \Psi=0$, where $\Psi$ is the wave function. The first-class constraints restrict the physical Hilbert space to the gaugeinvariant sector

$$
H_{1}^{\prime \prime} \Psi_{\text {phys }}=0, \quad H_{2}^{\prime \prime} \Psi_{\text {phys }}=0 .
$$

The general solution of (36) has the following form:

$$
\Psi_{\text {phys }}=f\left(\lambda, x^{2}\right) \Psi(\Omega),
$$

where $f(x, \lambda)$ is some function, whereas $\Psi(\Omega)$ is wave function on the $\mathrm{N}$-sphere. In the physical Hilbert space, we make $H_{1}^{\prime \prime}, H_{2}^{\prime \prime}$ zero in $H_{0}^{\prime \prime}$. Taking into account (35) and (36) we immediately find the energy values

$$
E_{l}=\frac{\hbar^{2}}{2 R^{2}} l(l+N-1)
$$

and conclude that the quantum Hamiltonian for the multidimensional rotor is given by the pure Schrödinger operator without any boundary term.

\section{Concluding remarks}

Despite the success of Dirac's approach in studying singular systems, which is demonstrated by the wide number of physical systems to which this formalism has been applied, it is always instructive to study singular systems through other formalisms, since different procedures will provide different views for same problems, even for non-singular systems.

In the Hamilton-Jacobi formalism we have a set of partial differential equations to start with, and we construct the phase space using the integrability conditions of a set of total differential equations. In this formalism we have no distinction between the firstand the second- class constraints but the Dirac's consistency conditions are equivalent to the Hamilton-Jacobi integrability conditions.

In this paper the Hamilton-Jacobi formalism was applied to investigate the nonrelativistic particle moving on a hypersurface in a curved manifold and we found the 
same set of constraints as by using Dirac's approach. In the case of the multidimensional rotator we have eliminated the non-physical degrees of freedom transforming the Cartesian coordinates into curvilinear ones.For the three- dimensional rotator a canonical transformation was performed in order to find the physical degrees of freedom and the action was calculated.

As pointed in [10] the energy spectrum of the multidimensional rotator obtained by Dirac's quantization method must be rejected because it is physically incorrect. Using the fact that $\lambda$ is a gauge parameter in the Hamilton-Jacobi formalism we found the same result as in [10]. The quantum Hamiltonian of the multidimensional rotor is given by pure the Schrödinger operator without any boundary term.

As a further step we will apply this method to non-Abelian gauge theory and gravity. This programme is under investigation, and this article is the first step in this direction.

\section{Acknowledgements}

DB would like to thank M. Olshanetsky for useful discussions. This paper is partially supported by the Scientific and Technical Research Council of Turkey.

\section{References}

[1] Dirac P A M 1964 Lectures on Quantum Mechanics (Yeshiva University, New York, N. Y. )

[2] Hanson A , Regge T and Teitelboim C 1976 Constrained Hamiltonian systems (Academia Nationale dei Lincei, Rome)

[3] Sundermeyer K 1982 Constrained Dynamics Lecture Notes in Physics vol. 169 (Springer-Verlag, New-York )

[4] Henneaux M and Teitelboim C 1992 Quantization of Gauge Systems (Princeton University Press, Princeton, New Jersey)

[5] Shabanov S 2000 Phys. Rep. 3261

[6] Woodhouse N M J 1992 Geometric Quantization Second Edition (Clarendon Press, Oxford),

Souriau J M 1997 Structure of Dynamical Systems (Birkhäuser, Boston), Kirilov A A 1990 Geometric Quantization in Dynamical Systems IV: Symplectic Geometry and its Applications, Arnold V I and Novikov S I Eds. Encyclopaedia Math. Sci. IV (Springer, New York)

[7] De Witt B 1952 Phys. Rev. 85653

De Witt B 1957 Rev. Mod. Phys. 29377 
[8] Marinov M S 1980 Phys. Rep. 601

[9] Podolski B 1928 Phys. Rev. 32812

[10] Kleinert H and Shabanov S V 1997 Phys. Lett. A 232327

[11] Homma T, Inamoto T A and Miyazaki T 1990 Phys. Rev. D 422049

[12] Omote M and Sato H 1972 Prog. Theor. Phys. 471367

[13] Saa A 1997 Class. Quant. Grav. 14385

[14] Bertolami O 1991 Phys. Lett. 55154

[15] Güler Y 1987 Nuovo Cimento B 100267

[16] Güler Y 1992 Nuovo Cimento B 1071389

[17] Güler Y 1992 Nuovo Cimento B 1071143

Güler Y 1998 Nuovo Cimento B 113893

[18] Carathéodory C 1967 Calculus of Variations and Partial Differential Equations of the First Order Part II, (Holden-Day )

[19] Kastrup H A 1983 Phys. Rep. 101 no.1-2 3

[20] Pimentel B M and Teixeira R G 1996 Nuovo Cimento B 111841

[21] Pimentel B M and Teixeira R G Nuovo Cimento B 1998113 B 805

[22] Pimentel B M , Teixeira R G and Tomazelli J L 1998 Ann. Phys. 26775

[23] Baleanu D and Güler Y 1999 Nuovo Cimento B 114 no.6 709

[24] Baleanu D and Güler Y 1999 Nuovo Cimento B 114 no.9 1023

[25] Baleanu D and Güler Y 2000 Nuovo Cimento B 115 no.1 25

[26] Faddeev L D and Shatashvili S I 1986 Phys. Lett. B 167225 , Batalin I A and Fradkin E S 1986 Phys. Lett . B . 180 156,

M. Henneaux Phys. Lett. B 315 (1993) 283

[27] Rajaraman R and Mitra P 1990 Ann. Phys. (N. Y.) 203 137, 157, Annishetty R and Vytheeswaran A S 1993 J. Phys. A 265613

[28] E. Rabei and Y. Güler 1992 Phys. Rev. A 463513

[29] Prokhorov I V and Shabanov S V 1991 Sov. Phys. Uspekhi. 34108

[30] Batalin I A and Fradkin E S 1987 Nucl. Phys. B . 279514 